\title{
Research Square
An Effectiveness and Cost-Estimation Model for Deploying Assistive Technology Solutions in Elderly Care
}

Petra Maresova ( $\square$ petra.maresova@uhk.cz)

University of Hradec Kralove https://orcid.org/0000-0002-1218-501X

Lukas Rezny

University of Hradec Kralove Faculty of Informatics and Management: Univerzita Hradec Kralove Fakulta informatiky a managementu

Petr Bauer

University of Hradec Kralove: Univerzita Hradec Kralove

Oluwaseun Fadeyia

University of Hradec Kralove: Univerzita Hradec Kralove

Olaniyi Eniayewu

Swansea University

Sabina Barakovic

University of Sarajevo University Tele-Information Centre: Univerzitet u Sarajevu Univerzitetski tele-informaticki centar

Jasmina Husic

University of Sarajevo Pedagogical Academy: Pedagoska akademija Univerziteta u Sarajevu

\section{Research}

Keywords: assistive technology, activities of daily living, model, costs, efficiency

Posted Date: January 10th, 2022

DOI: https://doi.org/10.21203/rs.3.rs-1226225/v1

License: (c) (1) This work is licensed under a Creative Commons Attribution 4.0 International License. Read Full License 


\section{Abstract \\ Background}

Deployment of modern assistive technologies is one of the major trends contained in the strategies of developed countries. However, the use of technology in households is not yet a common practice. The aim of this paper is present a model for assessment of selected smart device solutions in elderly care and the evaluation of overall care costs. The model provides the optimal set of devices for different target groups in terms of financial savings.

\section{Methods}

The model uses demographic projections taken from Eurostat for EU countries and the disability incidence from the annual report of the Ministry of Labour and Social Affairs of the Czech Republic as an input. The model was implemented in the software Stella Professional dedicated to system dynamics modelling including a web interface and is accessible online.

\section{Results}

In relation to the combination of five assistive devices for the elderly, the optimal solution, the cost savings are $37.8 \%$ or 182 billion CZK), cumulatively in the simulated time period 2021-2060. Out of the five available assistive devices, up to three - UpWalker, Jaco robotic arm and Poseidon - were employed by the model for different target groups.

\section{Conclusion}

According to the performed analysis the assistive technologies proved a significant potential to maintain the quality of life of elderly and lessen the burden on public budgets. With respect to the ongoing demographic transition, the need to employ smart device solutions should further increase and their price could decline with increasing scale of production and overall advancement in technology.

\section{Introduction}

Changes in demography across many developed countries and massive technological improvement have brought forward new paradigm regarding population vulnerability.

Since there is insufficient research on deployment of smart technological solutions, data are limited on the potential health and socioeconomic benefits of assistive technology. Although assistive technology is recognized as a human right, only $5-15 \%$ of the population that need it have access to it (Blauwet et al., 2020). However, access to assistive technology can have many positive effects in terms of health and socioeconomic benefits. Assistive technology supports the desire for aging in place and independence of elderly people allowing them and their caregivers to improve their quality of life. In order to achieve the objective of assistive technology to be developed in a highly accessible, compassionate and ethical way, it is important for the technologies to be usable and affordable (Vollmer Dahlke \& Ory, 2020). In combination with other critical efforts to help elderly people and their caregivers, assistive technology can open up potentials that have too long remained neglected in global health and socioeconomic discussions (Blauwet et al., 2020).

Since assistive technology has been assigned the low priority on the research agenda, the objective of this study is to provide new perspective to relevant stakeholders on need to extend their efforts to provide assistive technology. The basic objective is to evaluate and show specificity to the costs and associated benefits of providing smart technological solutions for seniors. The model is presented to calculate the benefits of economic improvements in quantitative terms, i.e. investment needed to build up delivery of assistive technology and costs associated to its usage. The intention is to conduct costeffectiveness analysis on the deployment of smart technological solutions for seniors.

In order to answer the posed question, this paper uses the methodology which is described in the following structure: Section 2 provides theoretical background covering description of activities of daily living (ADL) and instrumental activities of daily living (IADL) as well as assistive technologies. This section also talks about cost-effectiveness of assistive technologies, but covers them as well from medical and technical point of view. Further on, section 3 provides the methodology used to conduct this research and covers description of the study design, model description, together with data from Czech facilities used to verify the model. Also, this chapter explains the technology selection. Section 4 and 5 give the obtained results and discuss them. Section 6 concludes the paper and provides directions for future research.

\section{Theoretical Background}

Assistive technologies for seniors cannot be considered apart from present and upcoming concept of smart aging. Smart aging promotes well-being of aging population by seamless information technology, medical systems and devices, bio-technology, and robotics. According to Song et al. (2018), smart aging literature in terms of components and analysis covers three topics: behavior and social aspect, technologies, and medical care, but in terms of research activities, only behaviour and social aspect and medical care are covered. Technologies, therefore, require more intense effort in terms of research activities.

In addition, the existing research activities related to technologies need to be investigated in terms of senior satisfaction with them (Barakovic Husic et al., 2020). The effectiveness of future assistive technology solutions should be considered in various terms, i.e., satisfaction, medical contribution, but from the 
economic point of view as well. Assistive technologies should be incorporated into this idea of multidimensional and multidisciplinary personalized approach to senior individuals, i.e., smart aging. In fact, the ultimate goal of assistive technologies in terms of elderly people should be to improve their quality of life (QoL). Thus, assistive technology solutions in order to be effective need to contribute to various aspects of senior's life, i.e., material living conditions, health, education, productive and valued activities, governance and basic rights, leisure and social interaction, natural and living environment, economic and physical safety, and overall experience of life (Eurostat, 2021). In other words, these technologies should be designed to be personalized and satisfy multiple smart aging components which in combination contribute to multiple QoL dimensions, which then jointly accomplish the goal of senior's QoL (Barakovic Husic et al., 2020) .

Besides this QoL point of view on effectiveness of assistive technologies, which is inclusive per se since QoL covers multiple aspects, one should address medical and healthcare aspect specifically. One of the contributions, if technology is considered effective, would simply be improved health condition of elderly adult. Achieving that can be made through direct solutions intended for elderly adults but also solutions for assisting their health carers. Many ICT solutions currently proposed are targeted to prolong and give support the independence of older adults and provide help for professional and informal carers (Pal et al., 2018).

On the other hand, assistive technologies are a driver of increasing healthcare costs. In order to provide adequate response to that concern, it is necessary to understand the effectiveness of assistive technology being complemented by information about its costs, i.e. cost-effectiveness analysis. It is powerful analytical instrument used to compare costs of interventions based on patient outcomes (Sanders et al., 2019). Costs of interventions are related to products and services associated with assistive technology provision and maintenance, including the expenditures for evaluation of users, selection of devices, adaptation of devices to users' needs, education for their use and keeping them in operating conditions (Fuhrer, 2007).

Understanding the costs of interventions is crucial to transferring interventions from the research setting into the society. Cost-effectiveness analyses have been completed on healthcare interventions but have found inconsistent results (Bowes et al., 2013). For example, de Batlle et al. (2021) reported that implementation of various assistive technologies reduced the heath costs and was cost-effective. On the other hand, (Howard et al., 2021) found that assistive technology was not either effective or cost-effective. However, cost-effectiveness analyses on assistive technologies have yet to be completed (Shaw et al., 2021).

\section{Methodology}

\subsection{Design of study}

The aim of the paper is to present the results of the calculation of the effectiveness of the deployment of a smart technological (assistive technology) solution for seniors, which will enable the saving of human resources, which are in short supply in the field of care for the elderly. The setup of the model was prepared in the following steps:

- Specification of activities with which seniors are normally assisted, using the characteristics of ADL and IADL

- Specification of existing technological solutions and in connection with IADL

- Characteristics of the population in developed countries

- Finding evidence of costs related to IADL

- Setting the links of the model so that it is possible to determine under what conditions the deployment of the technology is effective.

Different scenarios were created in the model to answer the following questions:

- Which price allows to cover the costs associated with the new technology solutions?

- Where is the break-even point for chosen solutions in the market?

\subsection{Model description}

The model was implemented in the software Stella Professional, version 1.9 developed by ISEE Systems, with the hope of modelling the dynamic behaviour of the market associated with the increasing adoption of new solutions for ADL support. Due to the lack of reliable data, this part was eventually skipped, and the prices of assistive devices are exogenous variables along with the rest of input data.

In its current version, the model evaluates the most economical combination of available assistive devices for each age group and disability class (together referred to as category) based on their price and capability, before computing the overall expenses for provided care on national level and potential savings having adopted suggested solution.

The model uses demographic projections taken from Eurostat with age cohorts ranging from 0 to $100+$ by one year. These data are then partially summed into age groups $0-17,18-59,60-69,70-79,80-89,90+$. The population data for Czech Republic and other EU countries are available as an input.

From the annual report for 2019 of the Ministry of Labour and Social Affairs of the Czech Republic, the total number of people receiving disability compensations was obtained for the above age groups and the four disability classes (I to IV). The compensations are provided to people who, due to the

Page 3/15 
enduring health problems, require the assistance of another person in performing the basic ADLs. Based on the number of activities where aid is required, the disability classes are defined by the law to determine the value of the compensation.

The relative fractions (percentage) of people for each disability class and each age group are considered constant throughout the simulation. Multiplying them by the population data for given year, we compute the total number of people receiving care in each category (Table 1).

Table 1 - Number of people per age group and disability class (in thousands) for Czech Republic.

\begin{tabular}{|llllllll|}
\hline Class & $0-17$ & $18-59$ & $60-69$ & $70-79$ & $80-89$ & $90+$ & CZ total \\
\hline I & 12.2 & 16.4 & 12.7 & 21.9 & 31.8 & 7.7 & 102.8 \\
II & 8.8 & 20.2 & 14.3 & 25.2 & 36.2 & 12.4 & 117.0 \\
\hline III & 5.6 & 16.7 & 9.7 & 17.7 & 26.7 & 12.6 & 89.0 \\
IV & 4.5 & 11.7 & 4.3 & 8.9 & 15.3 & 8.9 & 53.5 \\
\hline CZ total & 31.1 & 65.0 & 40.9 & 73.8 & 110.0 & 41.6 & 362.4 \\
\hline
\end{tabular}

Source: Annual report of Ministry of Labor and Social Affairs for 2019 (MPSV, 2020)

The required hours of care per person for each category and the ADLs of interest (WC, hygiene, food, movement indoor/outdoor) were estimated by averaging the data provided by home-care services (description in chapter 3.3.1). This yields a 3D array indexed by the age group, disability class and ADL which is then used to calculate the total hours of care and the overall expenses by means of built-in array functions, including element-wise arithmetic and partial sums (along one or more dimensions).

Assistive devices are capable to replace a portion of overall hours provided by caregivers. Each device is described by a set of coefficients between 0 and 1 representing its efficiency (the approximate fraction of care hours it can handle) for given ADL. The economical parameters of the device are its price and its lifetime (in total hours of service.

If multiple devices have a non-zero value for the same ADL, the maximum of these values is used. Due to overlapping capabilities of devices, the real value of a device drops significantly, when a device with similar characteristics is already part of the combination.

The amount of replaceable care hours and the corresponding financial savings are computed for all combinations, yielding a winner in each category. As an auxiliary value, the break-even costs are evaluated.

\subsubsection{Input fields}

Population (2D) [Simulation_Year; Age] - Eurostat population projection starting from 2019 by 1-year cohorts without the distinction of sex.

Disability_Incidence (2D) [Age_Group; Disability_Class] - The relative fraction of people in each disability class for given age groups.

SD_Efficiency (2D) [SD; ADL] - The efficiency of available smart devices. Values between 0 and 1 representing to which extent the given SD can replace a human caregiver for the respective ADLs.

SD_Lifetime (1D) [SD] - The expected lifetime in years for given SDs.

SD_Purchase_Cost (1D) [SD] - The purchase costs of given SDs.

Caretaker_Hourly_Wage - The average hourly wage of caretakers for country in question.

SD_Availability (1D) [SD] - Boolean array provided by the user indicating which SDs are available for deployment. SDs with zero value will not be included in the processed combinations.

Hours_of_Care_per_Person_and_ADL (3D) [Age_Group; Disability_Class; ADL] - The typical hours of care for each ADL required by a person in given age group and disability class.

\subsubsection{Computed Fields}

Max_Savings (2D) [Age_Group; Disability_Class] - Maximum achievable yearly savings per person in given category.

Best_Combo (2D) [Age_Group; Disability_Class] - The most economical combination of SDs in terms of overall savings for given category.

Best_Combo_Costs (2D) [Age_Group; Disability_Class] - Yearly costs of the winning combination.

Replaced_Hours_per_ADL (3D) [Age_Group; Disability_Class; ADL] - Hours of human care per person and ADL that can be saved using the winning combination of SDs.

Selected_Combo_Yearly_Costs - The actual yearly costs of the combination of devices selected by the user via the interface window. 
Combo_Breakeven_Yearly_Costs (3D) [Combo; Age_Group; Disability_Class] - Breakeven yearly costs of all available SD combinations for each category.

Total_Costs_without_SDs - The overall yearly costs of care provided by human caregivers.

Total_Savings - The overall yearly savings when deploying the most economical combination of SDs in each category.

Percentage_Savings - The relative savings over the entire simulation period.

\subsubsection{Sector description}

Demography Sector - The population data from Eurostat projections are summed into six groups. The result is multiplied by the disability incidence, yielding the overall number of people for each age group and disability class which is used as an input in the care sector (figure 1).

Based on projections for Czech Republic, the total number of people in the working age (cohort 18-59) is expected to drop from 5.98 million to 5.03 million by 2050 , whereas the $60+$ cohort is about to grow from 2.88 million to 3.81 million. The total number of people receiving disability compensations is expected to grow from 385 thousand to 609 thousand. These are preliminary results which depend on the long-term population changes and the actual percentage of people receiving care (figure 2).

SD_Evaluation Sector - Based on user input, all possible combinations of available SDs are examined. The combo efficiency for given ADL is taken as the maximum of the individual efficiencies of the comprising SDs. Multiplied by the yearly care hours per person in each category, this provides us with the total number of replaceable care hours for the considered combo. The associated yearly operating costs are then compared with the corresponding wages of a human caregiver, yielding the breakeven yearly costs and the potential savings. The winning combination differs between categories, as the more expensive solutions become viable with the increasing hours of required care. The yearly savings in terms of both hours and expenses are used as inputs in the care sector (figure 3).

Care Sector - Multiplying the average care hours per person and ADL by the total number of people in the respective category, we get the overall hours of unassisted care per category, and subsequently its cost. Applying the best available combination per category as given by the SD_evaluation sector, the modified care hours along with the associated costs are computed. The results are then summed over all categories, yielding the total yearly costs for both assisted and unassisted care, and finally the perceptual savings throughout the simulation period (figure 4).

\subsection{Data}

Data from selected social care facilities in the Czech Republic and selected technologies with a direct link to the monitored ADL actions are used for the specific setting and verification of the functionality of the model.

\subsubsection{Data from selected social care facilities in the Czech Republic}

In 2020, data were requested on care for the elderly, which takes place in their home environment. The data were provided by two facilities, with a total of 609 clients (of which 194 men, 415 women). The information provided below is: age, gender, degree of dependence (I-IV) according to (MPSV, 2020) legislation in the Czech Republic, diseases / restrictions. Furthermore, the provided services were recorded, which are: purchases, routine cleaning and maintenance of the household, water delivery, accompanying adults, one-time import / removal, assistance and support in serving food and drink, assistance in dressing, assistance in using the toilet, assistance in orientation in the space, assistance in moving to a bed or wheelchair, assistance in preparing and serving food, assistance in personal hygiene, assistance with basic hair and nail care, washing and ironing bed or personal laundry, preparation and serving of food and drink, preparation and serving food individually, renting compensatory aids (mechanical wheelchair, walker, toilet chair), regular cleaning, large cleaning, heating in the stove, massages, pedicure (irrelevant to us) (table 2).

Table 2 - Average hours of care per disability class and ADL (age group 18 -90+). Source: own research in home-care services 


\begin{tabular}{|c|c|c|c|c|c|c|}
\hline class & Hygiene & WC & \multicolumn{2}{|c|}{ Indoor movement } & Walks & Feeding \\
\hline \multicolumn{7}{|c|}{ Age group 18-59 } \\
\hline I & 0 & 0 & \multicolumn{2}{|l|}{0.07} & 0.84 & 78.32 \\
\hline II & 7.537 & 0 & \multicolumn{2}{|l|}{1.97} & 0 & 0 \\
\hline III & 25.71 & 0 & \multicolumn{2}{|l|}{7.78} & 10.58 & 36.81 \\
\hline IV & 14.25 & 36.08 & \multicolumn{2}{|l|}{0.6} & 0.63 & 168.87 \\
\hline \multicolumn{7}{|c|}{ Age group 60-69 } \\
\hline I & 1.43 & 0 & 2.89 & 0.39 & \multicolumn{2}{|r|}{0} \\
\hline II & 8.92 & 0 & 7.45 & 4.14 & \multicolumn{2}{|r|}{15.11} \\
\hline III & 34.77 & 0 & 17.59 & 8.9 & \multicolumn{2}{|r|}{12.16} \\
\hline IV & 8.91 & 0.067 & 16.66 & 3.91 & \multicolumn{2}{|r|}{33.74} \\
\hline \multicolumn{7}{|c|}{ Age group 70-79 } \\
\hline I & 9.58 & 0 & 9.98 & 2.14 & \multicolumn{2}{|r|}{4.07} \\
\hline II & 16.45 & 3.98 & 5.51 & 6.14 & \multicolumn{2}{|r|}{17} \\
\hline III & 53.61 & 11.11 & 32.6 & 3.67 & \multicolumn{2}{|r|}{32.23} \\
\hline IV & 43.88 & 0 & 30.97 & 2.76 & \multicolumn{2}{|r|}{30.88} \\
\hline \multicolumn{7}{|c|}{ Age group 80-89 } \\
\hline 1 & 13.268 & 1.5 & 8.61 & 2.55 & \multicolumn{2}{|r|}{0} \\
\hline II & 15.45 & 0.63 & 14.48 & 4.31 & \multicolumn{2}{|r|}{20.53} \\
\hline III & 48.69 & 3.07 & 62.03 & 6.46 & \multicolumn{2}{|r|}{43.04} \\
\hline IV & 81.38 & 15.61 & 65.55 & 3.3 & \multicolumn{2}{|r|}{63.18} \\
\hline \multicolumn{7}{|c|}{ Age group $90+$} \\
\hline I & 1.95 & 0 & 3.15 & 1.61 & \multicolumn{2}{|r|}{0.05} \\
\hline II & 20.46 & 0 & 24.9 & 7.58 & \multicolumn{2}{|r|}{10.46} \\
\hline III & 57.33 & 0.356 & 30.48 & 3.44 & \multicolumn{2}{|r|}{12.56} \\
\hline IV & 117.77 & 0 & 77.66 & 2.89 & \multicolumn{2}{|r|}{93.06} \\
\hline
\end{tabular}

The parameters by which these actions were categorized and subsequently also financially valued are: number of hours, number of actions, number of visits, amount of payment per year, distance travelled.

In terms of the prepared model, the above information is used in four main categories of activities, which are described by the sums of hourly subsidies for the corresponding actions:

- personal hygiene - help with personal hygiene, help with basic hair and nail care

- Toilet - help with using the toilet

- movement - help with orientation in space, help with moving to a bed or wheelchair, one-time import / removal, shopping and errands

- food (cooking and serving food)

\subsubsection{Rate of replacement of the caregiver with a smart device}

In order to link information about the caregiver's time spent with individual tasks with clients and the functionalities of technologies for seniors, a nonstandardized interview was conducted with seven people in the field of social care. The main task was to find out to what extent they consider the given technology to be beneficial for the given operation (Table 3) and to express this rate in percentages. The interviews took place in February 2021. The technology was introduced using a short video of the product, and the interview was conducted. Table 3 presents the averages of all responses in each cell.

Table 3 Individual SD efficiencies for considered ADLs. 


\begin{tabular}{|lllllll|}
\hline Device/ADL & Hygiene & Toillet & Indoor Movement & Walks & Feeding & Meal Preparation \\
\hline UpWalker & 17,5 & 17,5 & 65 & 55 & 0 & 22,5 \\
\hline Jaco Robotic Arm & 2,5 & 0 & 12,5 & 0 & 47,5 & 12,5 \\
\hline Poseidon & 67,5 & 0 & 0 & 0 & 0 & 0 \\
\hline Toyota Human Support Robot & 7,5 & 5 & 57,5 & 35 & 25 & 10 \\
\hline Honda Walking Assistive Device & 5 & 5 & 55 & 60 & 0 & 2,5 \\
\hline
\end{tabular}

Five technologies were selected for the case study, with a direct link to ADL activities, so that it was possible to express the link to the observed variables in the model. Description of these technologies is provided below:

UPWalker is a mobility and standing support roller, designed by Prostar Inc., a Delaware-based company. Jaco Robotic Arm Manufactured by Kinova, a robotics company based in Quebec Canada, the Jaco range of robotic arms are some of the most useful ADL wares today, and have been researched to improve psychosocial ability of users (Beaudoin et al., 2019). In the realm of intelligent hygiene management, the Poseidon shower system performs care functions of assisting patients with bathing. The Human Support Robot (HSR) developed by Japanese corporation Toyota in 2012 is identified as one of the company's commitment toward the country's ageing population (RobotIEEE, 2017). The robot can carry out several features including communication, picking, lifting, and a few other ADLs (Yamamoto et al., 2018). As part of its quest toward R\&D in innovative mobility, Honda corporation developed an assistive walking device.

\section{Results}

\subsection{Model Assumptions}

Presented model simulation results were obtained with the following parameter values. For caregivers wage, we have assumed gross wage rate corresponding to the Czech republic conditions - $250 \mathrm{CZK} /$ hour. Regarding device costs, we had data only for Upwalker and Honda Walking Assist device, for rest of the devices, we had to assume their costs along with device longevity (assumed to be 5 years of usage for all devices). Cost data are presented in the Table 4 below.

Table 4

Price estimate of devices

\begin{tabular}{|ll|}
\hline Device name & Purchasing price [CZK] \\
\hline UpWalker! & 6000 \\
\hline Jaco Robotic Arm & $25000^{*}$ \\
\hline Poseidon & $75000^{*}$ \\
\hline Toyota Human Support Robot & $50000^{*}$ \\
\hline Honda Walking Assist Device & 40000 \\
\hline * - Assumed costs & \\
\hline
\end{tabular}

Rest of the parameters used for simulation were presented previously as they are the part of model structure and modelling approach, e.g. ADL replacement rates in Table 4.

\subsection{Cost development analysis}

Given current demographic trends in Czech Republic's population, the expected costs for elderly care (work of caregivers) in disability categories I-IV are expected to rise from current 8,31 bil. CZK to 16,6 bil. CZK (nearly doubling) in 2060, not including costs for medication or other than wage costs of institutions providing care.

First, we have analysed savings potential of each device employed separately. Estimation for the usage of the UpWalker is widespread in disabled elderly population with the total estimated cost savings of $29 \%$, or 139 bil. CZK in total cumulated savings. Jaco Robotic Arm has also significant potential, expressed by the $14,2 \%$ of savings potential, or 68,5 bil. CZK of savings cumulatively. Toyota Human Support Robot potential is also interesting thanks to a wide range of possible addressable ADL needs, with the result of $8,9 \%$ of savings potential, or 43 bil. CZK of savings in total. Honda Walking Assist Device results is weaker thanks its high specialization across $A D L$ dimensions and relatively high assumed price with the result of $5,1 \%$ of estimated savings, or 24,6 bil. CZK in total, cumulatively. Poseidon, smart device capable of addressing Hygiene ADL needs results were the worst thanks to its high specialization and price, making it suitable for institutional use only (results were $2,5 \%$ of total savings potential, or 12,1 bil. CZK cumulatively).

\section{Full-scale solution (ALL devices)}

In the following analysis, we focus on optimal solution from the perspective of cost savings in the system. All presented devices are used for optimization (without the consideration of possible synergies in between employed smart devices), while each device can be attributed separately to each age cohort and disability class of elderly (Table 5). 
Table 5

- Smart device assignment in respective age cohorts and disability classes

\begin{tabular}{|c|c|c|c|c|c|c|}
\hline \multirow[b]{2}{*}{ Age Group } & \multirow[b]{2}{*}{ Disability Class } & \multicolumn{5}{|c|}{ Smart Device } \\
\hline & & Upwalker & Jaco & Poseidon & Toyota HSR & Honda WAD \\
\hline $18-59$ & 1 & & $x$ & & & \\
\hline $18-59$ & 2 & & & & & \\
\hline $18-59$ & 3 & $x$ & $x$ & & & \\
\hline $18-59$ & 4 & $x$ & $x$ & & & \\
\hline $60-69$ & 1 & & & & & \\
\hline $60-69$ & 2 & $x$ & & & & \\
\hline $60-69$ & 3 & $x$ & & & & \\
\hline $60-69$ & 4 & $x$ & $x$ & & & \\
\hline $70-79$ & 1 & $x$ & & & & \\
\hline $70-79$ & 2 & $x$ & & & & \\
\hline $70-79$ & 3 & $x$ & $x$ & & & \\
\hline $70-79$ & 4 & $x$ & $x$ & & & \\
\hline $80-89$ & 1 & $x$ & & & & \\
\hline $80-89$ & 2 & $x$ & & & & \\
\hline $80-89$ & 3 & $x$ & $x$ & & & \\
\hline $80-89$ & 4 & $x$ & $x$ & & & \\
\hline $90+$ & 1 & & & & & \\
\hline $90+$ & 2 & $x$ & & & & \\
\hline $90+$ & 3 & $x$ & & & & \\
\hline $90+$ & 4 & $x$ & $x$ & $x$ & & \\
\hline
\end{tabular}

Maximum realisable cost savings are achieved with predominant usage of UpWalker and Jaco robotic arm. Poseidon, smart device capable of addressing elderly ADL Hygiene needs is employed in optimal solution only in the oldest age cohort with highest dependability.

Usage of concrete combination of devices is given by breakeven price, which is price of work of caregiver given by his/her wage and combination of $A D L$ needs in given age group and disability class which he/she needs to serve. Breakeven prices calculated in this way are depicted in Figure 5 below.

Unique combination of devices depicted in Table 5 need to suffice two conditions - price of their usage (purchasing price/lifetime) has to be lower than the work of caregiver and they have to supply needed ADL replacement (that is why Upwalker is typically not use in the first disability class despite its low cost).

The highest cost (49,2k, first line in figure 5) has the combination of devices Upwalker-Jaco-Poseidon, which is used only once in the model. Combination of Upwalker-Jaco is used 7x times in total (see Table 5) with total price of 6,2k (second line in figure 5) yearly. Upwalker alone is employed 8x times by the model, thanks to it low yearly price of usage equal to $1,2 \mathrm{k}$.

Optimal solution realisable cost savings are, $37,8 \%$ of total (or, 182 bil. CZK), cumulatively in the simulated time period of $2021-2060$, their expected time development is in the Figure 6.

\section{Sensitivity analysis}

Since we could not obtain pricing data regarding Poseidon and Toyota HSR, our estimates could be questioned. Thus we have employed sensitivity analysis where prices of aforementioned devices were varied in the range of $+-50 \%$ (42,5 thousand to 107,5 thousand CZK for Poseidon, 25 thousand to 75 thousand CZK for Toyota HSR).

Obtained results showed robustness of the model to this uncertainty as percentage savings varied only from $37,6 \%$ to $39,8 \%$, a mere $2,2 \%$ difference. Figure 7 shows this in absolute numbers.

The discrepancies was caused solely by Poseidon, which was employed twice with its lowest cost. Thus we can conclude that in the context of presented model, Poseidon and Toyota HSR can be sufficiently replaced by different, cheaper devices or their usage by elderly at home will probably never be economical. 


\section{Discussion}

Firstly, the results indicate that smart assistive technology devices inevitably are to be seriously considered as a way to mitigate challenges and issues of the aging population in this particular country, EU, and worldwide. This is no surprise given that they have been recognized in numerous strategic plans and projects (Blauwet et al., 2020; Nierling \& Maia, 2020; WHO, 2019, 2021) since the challenges with senior population are present almost everywhere. The second point to discuss is that one has to find the way to solve two parallel social trends and challenges with the assistive technologies worldwide: aging population and lack of healthcare workers. Smart assistive technologies should be accepted and supported not due to low prices and cost-savings given that the prices might go down with time, but because of labour shortages (Maresova, Hruska, et al., 2020; Maresova, Prochazka, et al., 2020) and increased number of seniors that need to be cared for (WHO, 2021). The benefit of applying smart assistive technologies is seniors' centres would be even more profound than in home environment, especially if one takes into account pandemic trends.

Going from global to particular case and having analysed the cost saving attributes of the different ADL devices, it is clear that some devices may remain unaffordable for a reasonable portion of population of elderly or disable persons in the Czech Republic. The implication is that demand will likely increase for cheaper devices like UPWalker and Jaco assistive arms.

In our current study, we are focused solely on homecare for elderly without considering institutional setup of the care. We have used various types of devices, from mechanical devices that are easily available and cheaper to robotic devices that are under development.

Their simulated deployment was dated to start in 2021 and with end of simulation in 2060. In the model, after setting a combination of suitable devices, their prices and population structure, which depends mainly on the size of the target group, a calculation is run showing the possibility of cost-efficient replacement of caregivers' time spent with seniors. The calculation shows the possibility of potential savings and costs with respect to caregivers' salaries.

Specific settings for the combination of the five devices listed in this case study (Table 5), their ability to replace some of the caregivers' actions (Table 3 ) and their price settings yield the total savings of $37,8 \%$ (or 182 bil. CZK), cumulatively in the simulated time period of 2021-2060.

It is obvious that the usage of devices such as Poseidon or Toyota HSR would be much more cost-efficient in institutional setup. However, we lack data about the degree of replace ability of employee/caregivers services in institutional context, thus our model does not take it into an account. Therefore, results should be interpreted with this limitation in mind as even higher cost saving could be achieved in institutional setup.

Another limitation is the lack of data for production/sales cost for many devices in our selection. Even with currently sold devices, i.e. Honda walking assist device, where the price is known, it is likely that price will drop substantially in the case of successful market launch and increasing scale of production. In that case, the model results can be viewed as pessimistic, as they reflect current prices (or their estimates) only.

The model allows adjustments regarding price levels of various countries through the caregivers' wage. One can find that the approximate difference between Czech caregivers' wage and the wage in Germany, Norway, or Britain is approximately $30 \%$. With higher caregivers' wage, the model yields higher estimated cost savings than in Czech Republic with increased usage of smart devices for elderly assistance.

The model is currently using the Table 1 of elderly population ADL needs for Czech republic, while allowing to import population data and price level (through adjustable caregivers' wage) for another countries, which might distort results. While it would be trivial to turn the ADL needs table into yet another input quantity, the system of disability classes is rather unique for Czech Republic, which might require additional pre-processing of the respective data to fit into the model.

\section{Conclusion}

This paper aimed at finding the answer to cost-effectiveness of smart assistive technology for seniors. For that purpose, we have developed a model to calculate the effectiveness of applying the technology and conducted a case study with five assistive devices in the Czech Republic. The contributions of the paper are three-fold. Firstly, we proposed a model which may be used in other countries as well for the similar research activities which, as discussed earlier, are not represented to a great extent. Second, we recognized the need for further studies to treat the effects of assistive technologies implementation on both individual and national level. Third contribution is the conclusion that the assistive technologies cannot be considered only in terms of financial effectiveness, but much broader, given that the benefits they bring can be short- and long-term, and direct or indirect.

The benefits of assistive technologies are reflected in health improvements, social inclusion, and economic returns. Assistive technologies improve the health and wellbeing of the elderly, but also increase access to wider healthcare services. It facilitates greater inclusion of elderly people in society by lowering the risk of loneliness and encourage a sense of belonging. Providing assistive technology to elderly people will increase the rates of employment and productivity, by helping to overcome the challenges of ageing, thus allowing to work fully until retirement. In total, including smart assistive technologies in our everyday lives should lead to improvement of QoL directly, for the actual users,or indirectly by lowering the burden on the caregivers.

The assistive technologies are not only cost-efficient in terms of actual care costs, but their long-term impact on QoL of users, along within their potential to deal with population ageing and the associated healthcare provider shortage are highly beneficial to the society as a whole. In order toreach some of the benefits, the countries should improve the access to assistive technologies by taking specific actions, such as public support, policy and legislation changes, research and development support, etc. These activities will require the inclusion of all stakeholders (e.g., public sector, private sector, donators, etc.) and various research methods in order to provide comprehensive vision and priorities for resources.

\section{Declarations}


Not applicable

Consent for publication

Not applicable

Availability of data and material

The datasets used and/or analysed during the current study are available from the corresponding author on reasonable request.

Funding:

This work supported in part by the LTC INTER COST, Evaluation of the Potential for Reducing Health and Social Expenses for Elderly People Using the Smart Environment, through the Ministry of Education, Youth and Sports, Czech Republic, under Project LTC18035; and in part by the project of Excellence, University of Hradec Kralove, FIM, Czech Republic.

Competing Interests:

The authors declare that they have no conflict of interest.

Authors' contributions

PM collected analysed and interpreted data of elderly, PM, SB, JH suggested design of the study were contributors in writing also . LR and PB prepared model. OF and OE made a major contribution in writing the manuscript. All authors read and approved the final manuscript.

\section{References}

1. Barakovic Husic, J., Melero, F. J., Barakovic, S., Lameski, P., Zdravevski, E., Maresova, P., Krejcar, O., Chorbev, I., Garcia, N. M., \& Trajkovik, V. (2020). Aging at Work: A Review of Recent Trends and Future Directions. International Journal of Environmental Research and Public Health, $17(20)$, E7659. https://doi.org/10.3390/ijerph17207659

2. Beaudoin, M., Lettre, J., Routhier, F., Archambault, P. S., Lemay, M., \& Gélinas, I. (2019). Long-term use of the JACO robotic arm: A case series. Disability and Rehabilitation. Assistive Technology, 14(3), 267-275. https://doi.org/10.1080/17483107.2018.1428692

3. Blauwet, C., Casey, C., Clarke, H., Aguilar, C. D., Ndopu, E., Nkosi, L., Obasanjo, O., Parsons, A., \& Shriver, T. (2020). The Case for Investing in Assistive Technology: The dramatic economic, health, and social benefits of assisting a billion people to live fulfilling and dignified live (p. 80). ATScale Global Partnership for Assitive Technology. https://static1.squarespace.com/static/5b3f6ff1710699a7ebb64495/t/5fbf5c44eaf37e3b64932e6c/160637653

4. Bowes, A., Dawson, A., \& Greasley-Adams, C. (2013). Literature review: The cost effectiveness of assistive technology in supporting people with dementia (p. 62). Dementia Services Development Trust. https://dspace.stir.ac.uk/bitstream/1893/18500/1/the_cost_effectiveness_of_assistive_technology_in_supporting_people_with_dementia_october_13.pdf

5. de Batlle, J., Massip, M., Vargiu, E., Nadal, N., Fuentes, A., Ortega Bravo, M., Miralles, F., Barbé, F., Torres, G., \& CONNECARE-Lleida Group. (2021). Implementing Mobile Health-Enabled Integrated Care for Complex Chronic Patients: Intervention Effectiveness and Cost-Effectiveness Study. JMIR MHealth and UHealth, 9(1), e22135. https://doi.org/10.2196/22135

6. Eurostat. Quality of Life (QoL). Available online: https://ec.europa.eu/eurostat/web/gdp-and-beyond/qualityof-life/data (accessed on 4 April 2020).

7. Fuhrer, M. J. (2007). Assessing the efficacy, effectiveness, and cost-effectiveness of assistive technology interventions for enhancing mobility. Disability and Rehabilitation: Assistive Technology, 2(3), 149-158. https://doi.org/10.1080/17483100701374355

8. Howard, R., Gathercole, R., Bradley, R., Harper, E., Davis, L., Pank, L., Lam, N., Talbot, E., Hooper, E., Winson, R., Scutt, B., Ordonez Montano, V., Nunn, S., Lavelle, G., Bateman, A., Bentham, P., Burns, A., Dunk, B., Forsyth, K., ... Gray, R. (2021). The effectiveness and cost-effectiveness of assistive technology and telecare for independent living in dementia: A randomised controlled trial. Age and Ageing, 50(3), 882-890. https://doi.org/10.1093/ageing/afaa284

9. Maresova, P., Hruska, J., Klimova, B., Barakovic, S., \& Krejcar, O. (2020). Activities of Daily Living and Associated Costs in the Most Widespread Neurodegenerative Diseases: A Systematic Review</p>. Clinical Interventions in Aging, 15, 1841-1862. https://doi.org/10.2147/CIA.S264688

10. Maresova, P., Prochazka, M., Barakovic, S., Baraković Husić, J., \& Kuca, K. (2020). A Shortage in the Number of Nurses-A Case Study from a Selected Region in the Czech Republic and International Context. Healthcare, 8(2), 152. https://doi.org/10.3390/healthcare8020152

11. MPSV 2020-129422. Statistiky př́spěvek na péči. (accessed on 4 April 2020).

12. Nierling, L., \& Maia, M. (2020). Assistive Technologies: Social Barriers and Socio-Technical Pathways. Societies, $10(2), 41$. https://doi.org/10.3390/soc10020041

13. Pal, D., Funilkul, S., Charoenkitkarn, N., \& Kanthamanon, P. (2018). Internet-of-Things and Smart Homes for Elderly Healthcare: An End User Perspective. IEEE Access, 6, 10483-10496. https://doi.org/10.1109/ACCESS.2018.2808472

14. RobotIEEE. (2017). Human Support Robot. https://robots.ieee.org/robots/hsr/

15. Sanders, G. D., Maciejewski, M. L., \& Basu, A. (2019). Overview of Cost-effectiveness Analysis. JAMA, 321(14), $1400-1401$. https://doi.org/10.1001/jama.2019.1265

16. Shaw, C. A., Williams, K. N., Lee, R. H., \& Coleman, C. K. (2021). Cost-effectiveness of a telehealth intervention for in-home dementia care support: Findings from the FamTechCare clinical trial. Research in Nursing \& Health, 44(1), 60-70. https://doi.org/10.1002/nur.22076

Page $10 / 15$ 
17. Song, I.-Y., Song, M., Timakum, T., Ryu, S.-R., \& Lee, H. (2018). The landscape of smart aging: Topics, applications, and agenda. Data \& Knowledge Engineering, 115, 68-79. https://doi.org/10.1016/j.datak.2018.02.003

18. Vollmer Dahlke, D., \& Ory, M. G. (2020). Emerging Issues of Intelligent Assistive Technology Use Among People With Dementia and Their Caregivers: A U.S. Perspective. Frontiers in Public Health, 8, 191. https://doi.org/10.3389/fpubh.2020.00191

19. World Health Organization. (2019). Global perspectives on assistive technology: Proceedings of the GREAT Consultation (p. 540). WHO. https://apps.who.int/iris/bitstream/handle/10665/330372/9789240000261-eng.pdf

20. World Health Organization. (2021). WHO and European Union's funded Health and Care Cluster collaborating on Digital and Assistive Technologies for Ageing webinar series. https://www.who.int/news/item/03-06-2021-who-and-european-union-s-funded-health-and-care-cluster-collaborating-on-digitaland-assistive-technologies-for-ageing-webinar-series

21. Yamamoto, T., Nishino, T., Kajima, H., Ohta, M., \& Ikeda, K. (2018). Human support robot (HSR). Association of Computing Machinery, 1-2. https://doi.org/10.1145/3214907.3233972

\section{Figures}

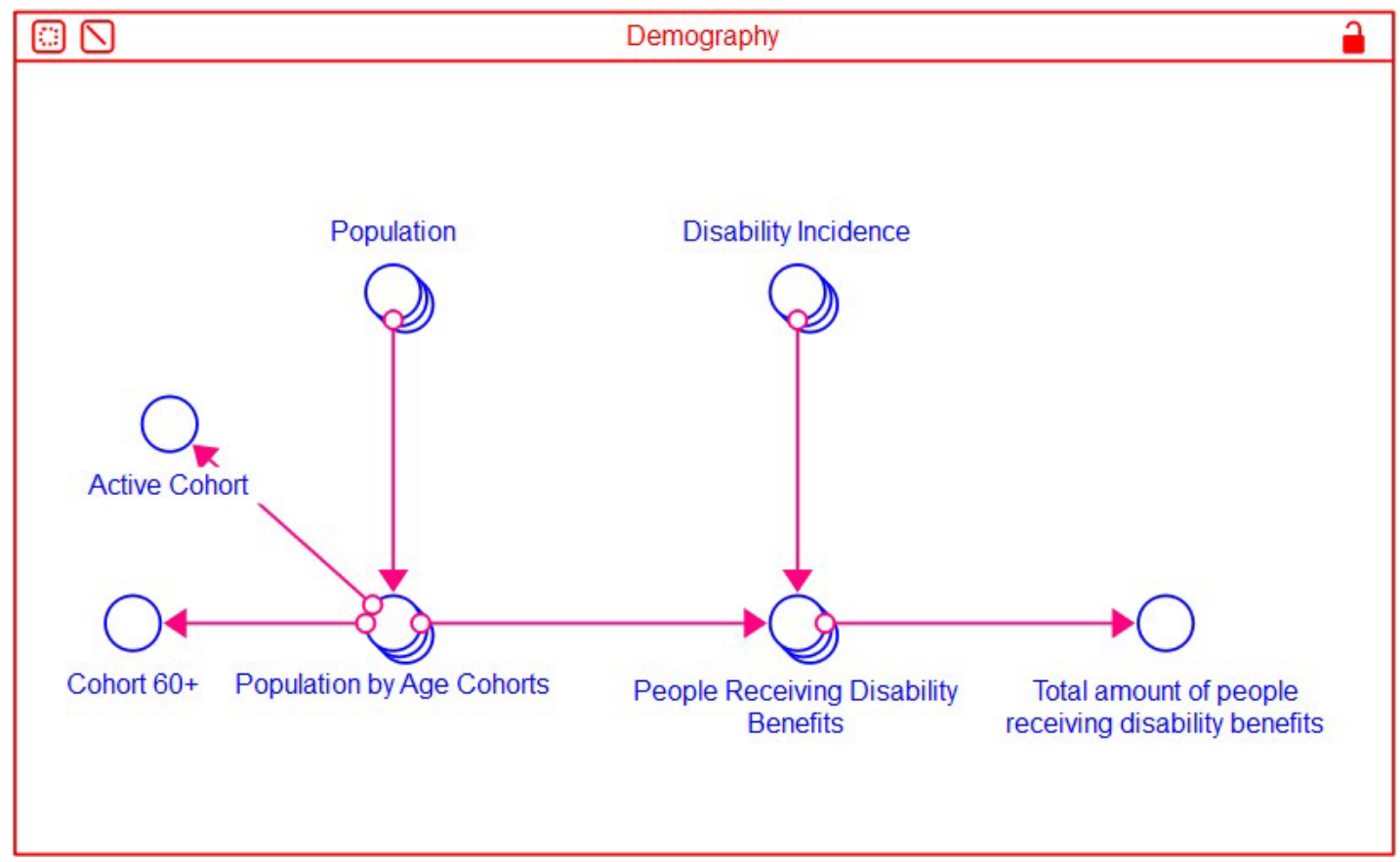

\section{Figure 1}

Demography sector 


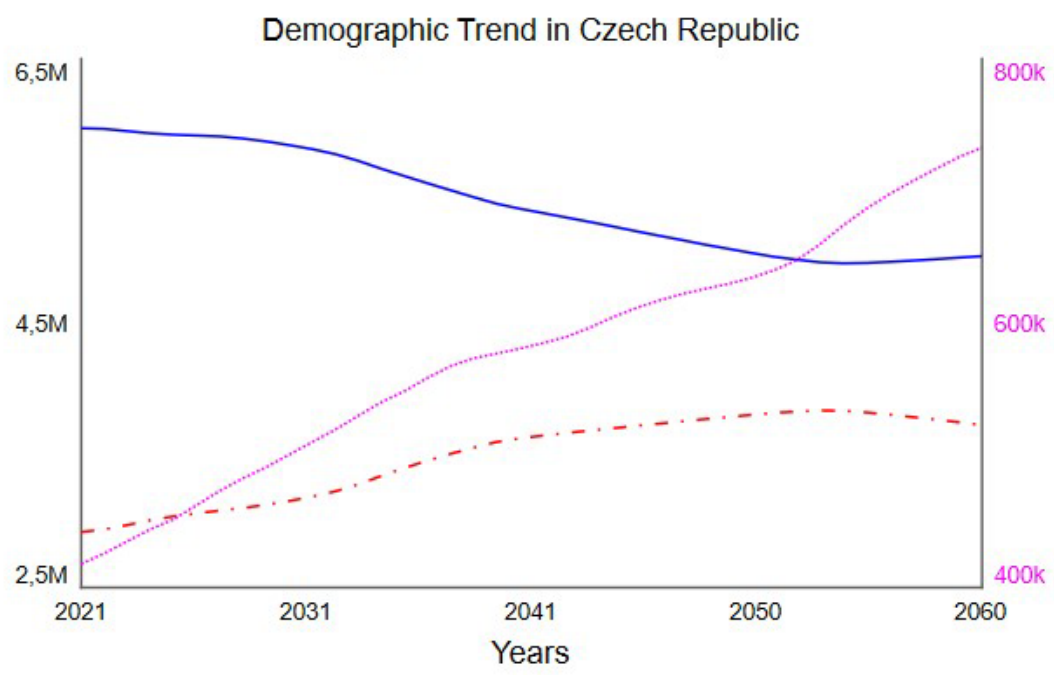

- Active Cohort

-. - Cohort 60+

........... Total amount of people receiving disability benefits

Figure 2

People in given age cohorts (left $Y$ axis) and total amount of people receiving disability benefits (right $Y$ axis)

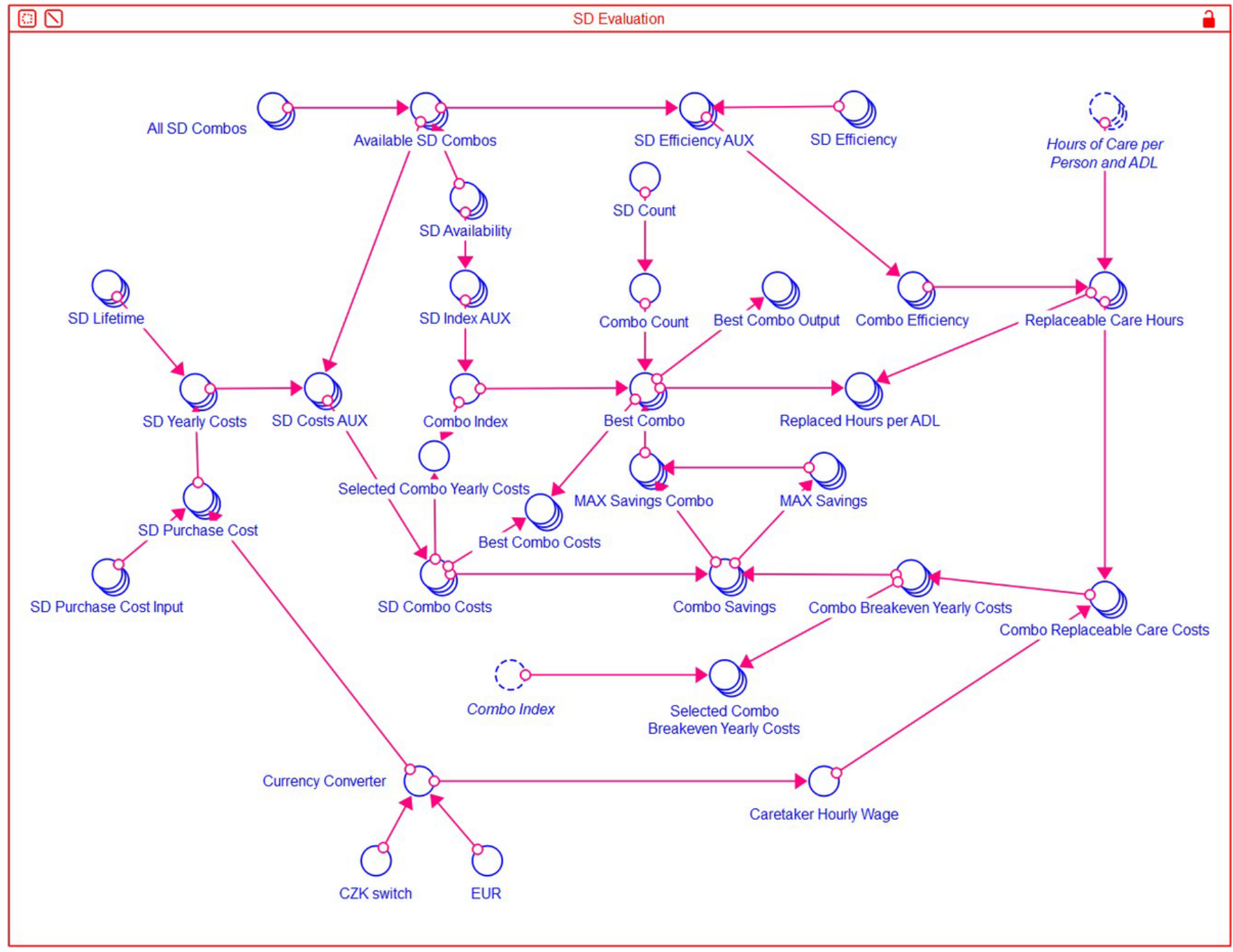

Figure 3

Page $12 / 15$ 
SD Evaluation process

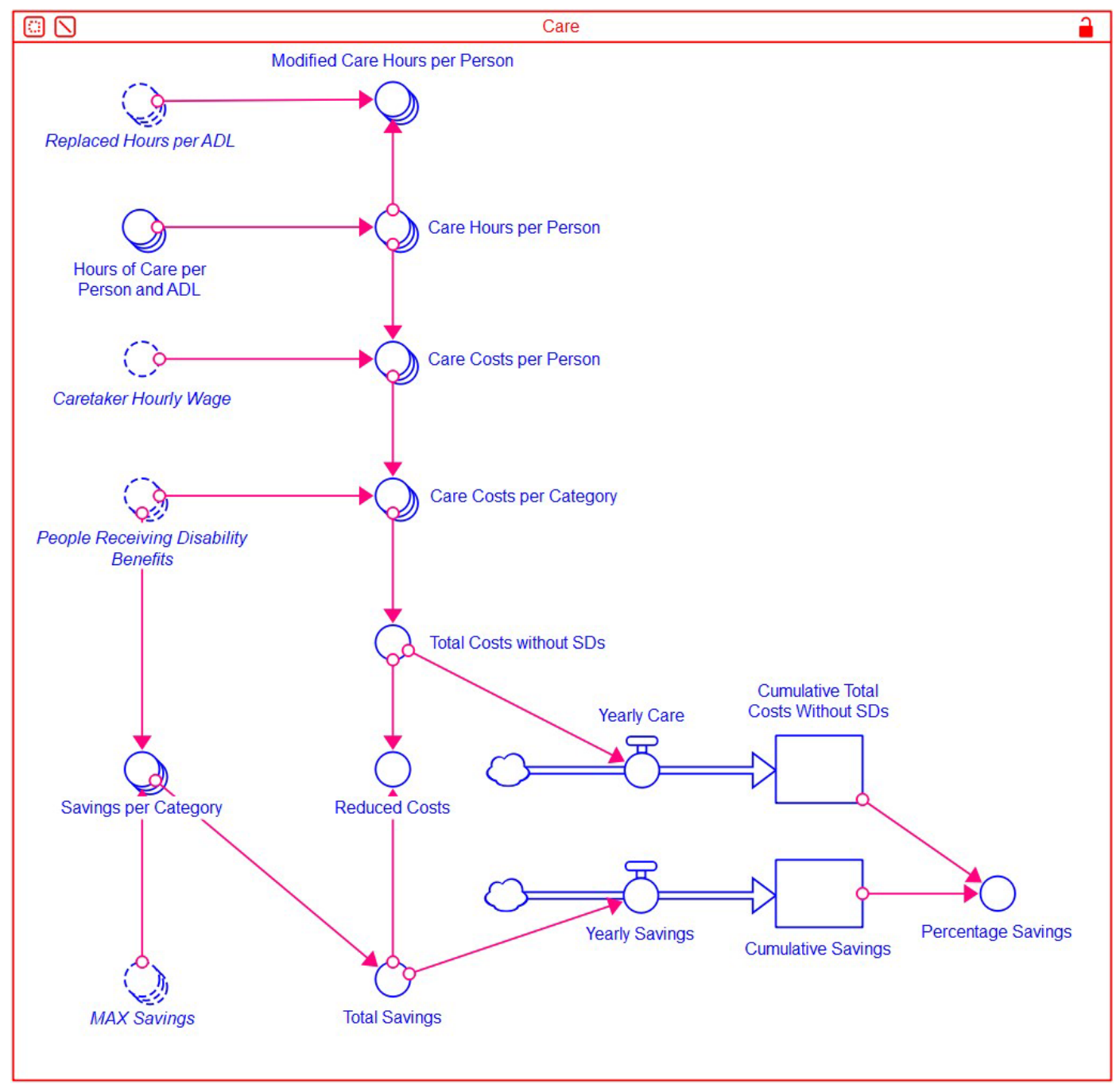

Figure 4

Care costs and savings 
Breakeven Estimated Yearly Cost for Selected Assistive Device Solution and Population Group

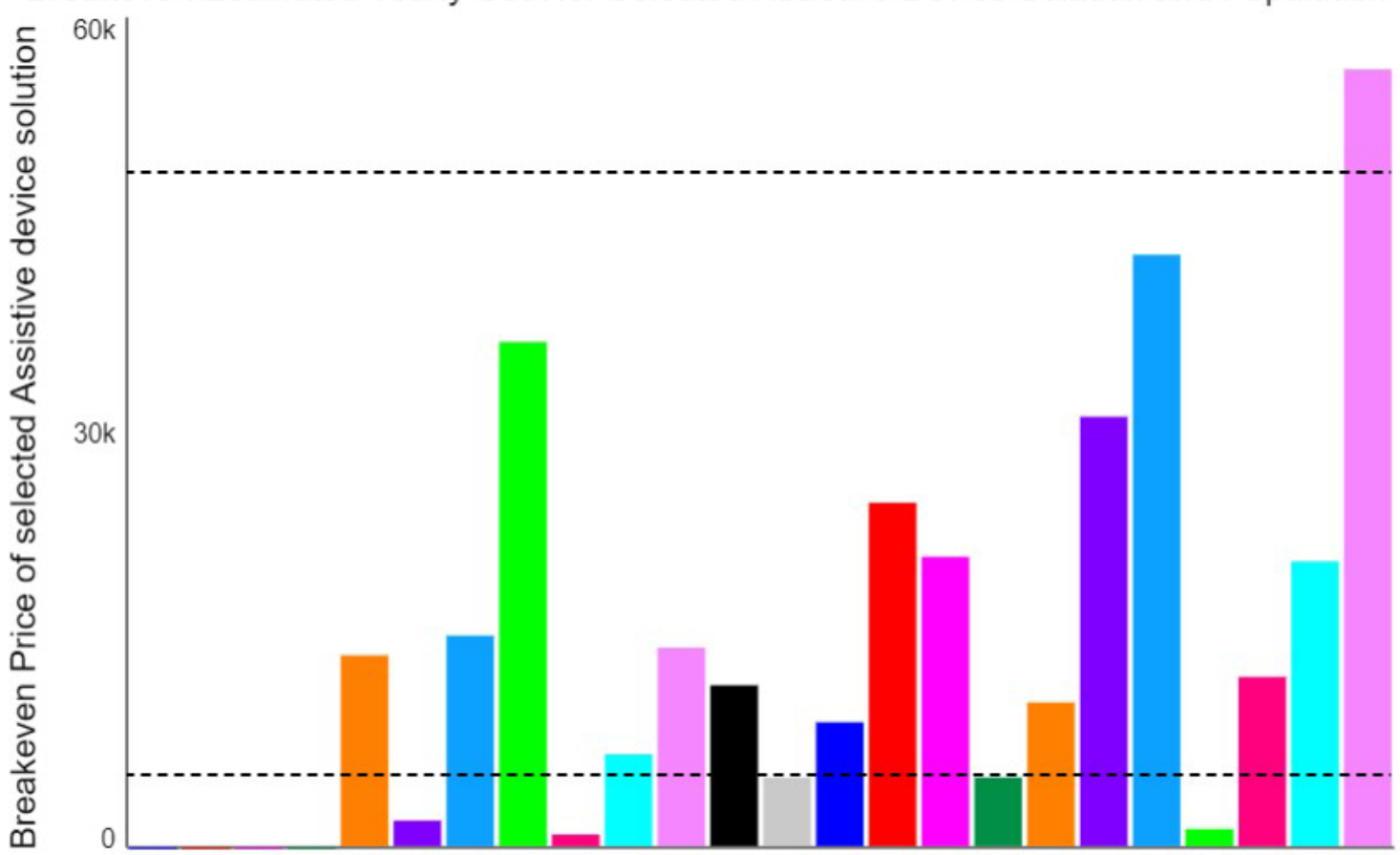

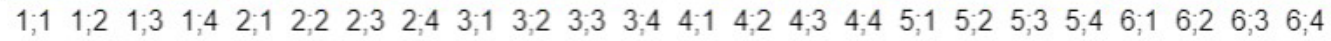

Group code; First Coordinate - Age Cohort; Second coordinate: Dependability class

\section{Figure 5}

Breakeven Yearly Price

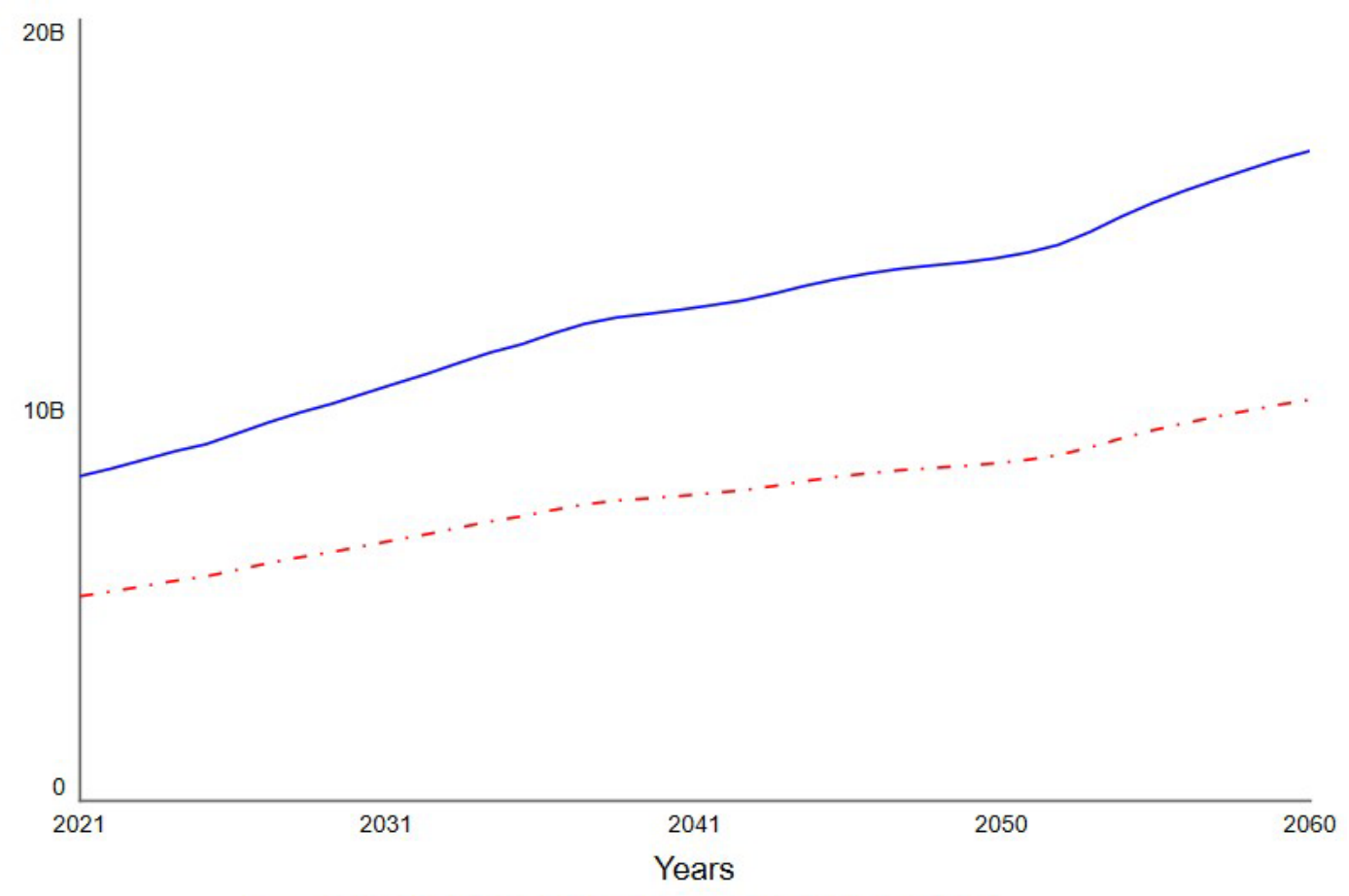

— Total Costs without SDs - - - Reduced Costs

\section{Figure 6}

Cost saving with the optimal device assignment across cohorts and disability classes 


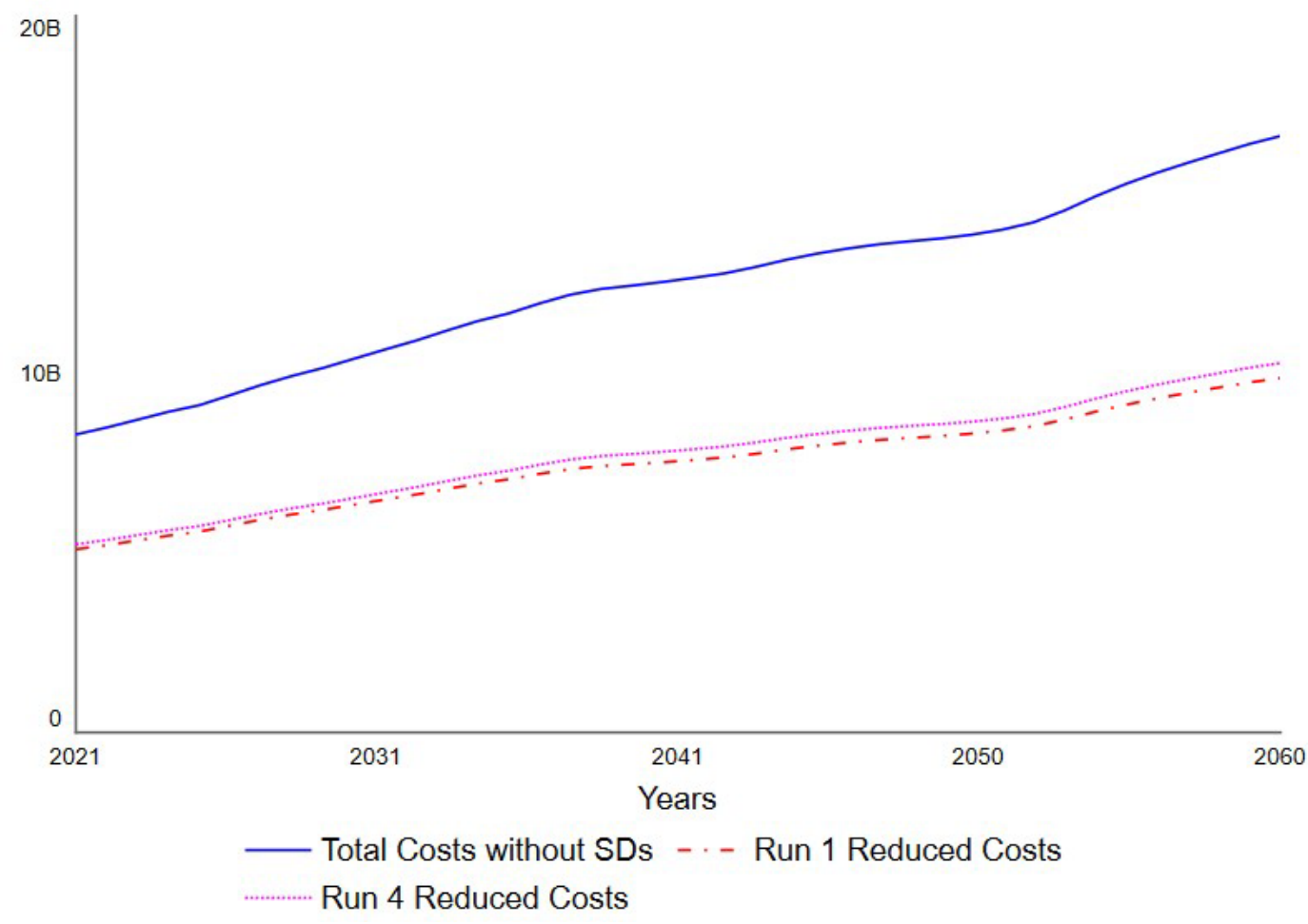

Figure 7

Sensitive analysis 